\title{
RÖDEL, Dieter, SCHNEIDER, Joachim, Strukturen der Gesellschaft im Mittelalter - Interdisziplinäre Mediävistik in Würzburg
}

Laurence Buchholzer

\section{OpenEdition}

\section{Journals}

Édition électronique

URL : http://journals.openedition.org/ifha/1460

DOI : $10.4000 /$ ifha. 1460

ISSN : 2198-8943

Éditeur

IFRA - Institut franco-allemand (sciences historiques et sociales)

Référence électronique

Laurence Buchholzer, «RÖDEL, Dieter, SCHNEIDER, Joachim, Strukturen der Gesellschaft im Mittelalter Interdisziplinäre Mediävistik in Würzburg », Revue de l'IFHA [En ligne], Date de recension, mis en ligne le 01 janvier 1998, consulté le 22 septembre 2020. URL : http://journals.openedition.org/ifha/1460 ; DOI : https://doi.org/10.4000/ifha.1460

Ce document a été généré automatiquement le 22 septembre 2020.

(CIFHA 


\title{
RÖDEL, Dieter, SCHNEIDER, Joachim, Strukturen der Gesellschaft im Mittelalter - Interdisziplinäre Mediävistik in Würzburg
}

\author{
Laurence Buchholzer
}

1 Au sein de l'université de Wurtzbourg, l'histoire médiévale fédère depuis une vingtaine d'années plusieurs groupes de réflexion interdisciplinaire. Sur les pas de Rolf Sprandel, ils marient heureusement l'histoire régionale et l'ouverture aux questionnements plus généraux.

2 Avec Strukturen der Gesellschaft im Mittelalter, les médiévistes de Wurtzbourg communiquent les résultats de leurs réflexions communes. Tous sont animés d'un même mot d'ordre: démonter la construction sociale médiévale; en mettre à jour les liens, la structure, les normes pour mieux comprendre cette société d'alors dans ses mouvements, ses jeux de force, ses mécanismes de régulation et de légitimation.

3 Sans fonder une nouvelle théorie de l'histoire sociale, leur recueil met en pratique, essentiellement dans l'espace franconien, quelques préceptes salutaires: circonspection envers les grands systèmes d'analyse de la société médiévale (noblesse, chevalerie, système judiciaire...), approche des composantes sociales en termes de positionnements, de relations et de construction... Le regroupement des articles s'efforce lui-même d'ouvrir de nouvelles perspectives qui tranchent avec les traditionnelles approches de la société en divisions "politiques«, »culturelles«, ou »religieuses«.

4 Un premier groupe de contributions analyse les relations entre les sexes au Moyen Age. Rudolf Weigand y étudie le recours aux tribunaux ecclésiastiques de Wurtzbourg et Ratisbonne pour affaires matrimoniales. La majorité des procès vise à la reconnaissance juridique d'une promesse de mariage non honorée. Par là se révèlent les progrès que doit encore accomplir l'Église au XVe s. dans l'encadrement des pratiques matrimoniales, les limites de l'autorité familiale, et la marge d'action des femmes 
abusées. Au travers des livres de fief des évêques de Wurtzbourg, Helga Möhring-Müller a étudié les pensions aménagées sur un fief épiscopal aux veuves nobles. Au cours du XVe s., les nobles ruraux prévoient pour leurs femmes des rentes de plus en plus importantes, bien au-dessus du seuil d'existence. Mais ils précisent aussi plus strictement les modalités de ces pensions, réduisant ainsi la part des contestations possibles au profit des héritiers. Dietrich Hischenbrett propose une analyse plus littéraire, en s'attardant sur le mariage de Ludwig der Springer avec Adélaïde, épouse du comte palatin Frédéric II de Goseck. En dépit de la variété des récits, l'histoire du meurtre de Frédéric II de Goseck, prélude au remariage d'Adélaïde, se transforme très nettement au XIVe s. en un roman d'amour courtois. Ce genre, par ses caractéristiques mêmes, offre l'avantage de ne pas avoir à justifier ni expliquer le meurtre originel, alors que la perpétuation biologique et l'ascension sociale de la lignée ont été garanties par le second mariage. Les comportements considérés aujourd'hui comme féminins ou masculins sont des constructions sociales qui peuvent remonter au Moyen Age. L'obéissance, la mesure, la réserve prescrites au XIIIe s. aux novices, qu'ils soient de sexe masculin ou féminin, sont prônées pour les seules filles par les traités d'éducation laïcs de la fin du Moyen Age. Doris Ruhe décrit ce processus et en recherche les vecteurs.

Plusieurs fois inscrit en filigrane des contributions précédentes, le rôle des gens d'Église motive explicitement les propos d'Edgar Hösch et Klaus Wittstadt. Au travers des modalités d'introduction du christianisme en Russie par Byzance et de la catégorie des "gens d'Église«, on peut mesurer l'impact des contacts culturels sur la formation de nouvelles structures sociales (Edgar Hösch). Plus classiquement, Klaus Wittstadt situe les évêques et les établissements réguliers de Wurtzbourg dans le courant pastoral et les efforts de réforme de la fin du Moyen Age.

6 Les groupes de recherche les plus féconds de Wurtzbourg portent, à n'en pas douter, sur le règlement des conflits, qu'il soit guerrier, amiable ou judiciaire. Horst Brunner exhume dans la littérature médiévale allemande les perceptions successives de la guerre, de la guerre de mission à la guerre absurde. Il rejoint ainsi un axe majeur des études générées par Rolf Sprandel: le monde des représentations, en ce qu'il peut avoir de générateur, de mobilisateur et de révélateur pour les groupes sociaux. Fort d'une telle perspective, Joachim Schneider met à jour les stratégies d'accroche de deux manifestes initiés par la petite noblesse franconienne aux temps de la lutte contre les Hussites. Jouant des allusions aux voyages en Prusse, aux tournois et joutes de cour pour drainer la noblesse franconienne, les diverses versions linguistiques des manifestes savent aussi présenter d'autres arguments pour atteindre une dimension supra-régionale et toucher les princes. On peut voir dans ces appels une ultime tentative d'ouverture de la petite noblesse franconienne avant qu'elle ne se cimente en un groupe autonome, aux intérêts spécifiques. Joachim Schneider rejoint par là les travaux de Joseph Morsel sur la noblesse franconienne. Mais ce dernier s'attaque ici à un thème récurrent de l'historiographie allemande, la faide. Tour à tour expliquée par des arguments psychologiques, judiciaires, économiques et politiques, la faide semble plutôt avoir servi la hiérarchisation et la cohésion sociales au sein de la petite noblesse. Elle devenait par là un signe essentiel de reconnaissance et de manifestation du statut noble. Pendant plus d'un siècle, au sein de leurs ententes et contrats, les princes et la chevalerie franconienne se sont disputés le droit de régler les conflits et la composition des tribunaux d'arbitrage. Constance Proksch suit l'évolution de ces querelles qui soudèrent la chevalerie franconienne. On a longtemps interprété le règlement des 
conflits médiévaux en raisonnant avec les termes du droit pénal moderne. C'est oublier qu'au Moyen Age, même des cas de meurtres pouvaient se régler hors des tribunaux. Sur la base de coutumiers franconiens, Dietmar Willoweit invite à une nouvelle lecture des sources judiciaires rurales. Les possibilités offertes par les autorités, le droit ou les coutumes pour résoudre les tensions étaient nombreuses. Leur inventaire et sa confrontation avec les choix opérés par les partis en conflits peuvent s'avérer fructueux.

7 Les contributions suivantes se consacrent à la seigneurie. Au XVe s., le diocèse de Bamberg bénéficie toujours d'obéances en nature, alors que se multiplient les taxes en argent. Dans une perspective comparatiste avec la Chine et le Japon, Kunio Nashiro montre qu'il ne s'agit pas d'une simple réaction seigneuriale, mais bien d'une adaptation aux nécessités économiques et monétaires du temps. L'étude des exploitations agricoles et de leur taille peut être un moyen d'approcher les structures sociales du monde rural médiéval. Helmut Jäger rappelle les difficultés inhérentes à une telle démarche, tandis que Dieter Rödel explore la structure de populations villageoises franconiennes aux XIV et XVe ss. Si les coutumiers ne témoignent guère de la hiérarchisation paysanne, les censiers wurtzbourgeois en donnent un aperçu détaillé, analysable au moyen de courbes de Lorenz. Peter Rückert nous ramène au temps des grands défrichements, de leurs initiateurs et de leurs entrepreneurs locaux. Les évêques eurent un rôle important de donneurs d'ordre, mais en Haute-Rhénanie, ils durent céder le pas devant des familles comtales, qui renforçaient sur le tard leur autorité seigneuriale. Le monde des villes est abordé plus brièvement. Walter Janssen témoigne des apports fructueux de l'archéologie et de l'iconographie pour l'histoire sociale urbaine. Cette société urbaine s'enrichit au XIVe s. d'une nouvelle catégorie professionnelle, les maîtres-canonniers. Transfuges de branches artisanales voisines, ils gagnent progressivement en renommée, en spécialisation et en organisation (Rainer Leng).

8 Avant de s'enquérir du regard que divers groupes sociaux portent sur elle, il n'est pas inutile de rappeler ce que signifiait au fond »la société« pour les hommes du Moyen Age. Norbert Richard Wolf s'attache à cette histoire du concept. Des croisés thuringiens et des marins méditerranéens firent au large de Chypre l'expérience de leurs différentes conceptions de la mort et de la solidarité. Jürgen Petersohn saisit l'occasion de leur conflit, au retour de la dépouille de Louis III de Thuringe, pour démonter les mécanismes de leurs mentalités. En Occident, les Juifs furent souvent ces autres, mis au ban, mais indispensables. Sur les pas de F. Graus, Klaus Arnold cherche à Wurtzbourg l'enchaînement éventuel entre Grande peste, flagellants et élimination des Juifs. La peur de l'épidémie seule aurait suffi à précipiter la fin de la communauté juive. Dans un article antithétique, fondé sur la même base documentaire des chroniques de Wurtzbourg, H. Peter Baum dément l'existence à Wurtzbourg d'un suicide collectif des Juifs et cherche les raisons de cette mystification.

9 L'histoire sociale médiévale propose un champ de sujets et de méthodes si large que l'on peut craindre en bout de course l'incompréhension et l'immobilisme. En privilégiant un questionnement transversal aux groupes sociaux, Strukturen der Gesellschaft a su contourner la menace et innover. 